\title{
Inhibition of mitochondrial and cytosolic calpain attenuates atrophy in myotubes co-cultured with colon carcinoma cells
}

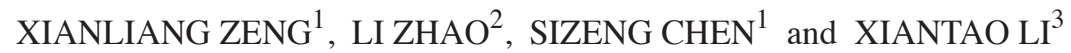 \\ Departments of ${ }^{1}$ Gastrointestinal Surgery, ${ }^{2}$ Dermatology and ${ }^{3}$ Vascular and Endovascular Surgery, \\ The First Affiliated Hospital of Fujian Medical University, Fuzhou, Fujian 350004, P.R. China
}

Received July 17, 2020; Accepted November 12, 2020

DOI: $10.3892 / 01.2020 .12385$

\begin{abstract}
Cancer cachexia is a life-threatening syndrome characterized by muscle atrophy. Cancer cachectic muscle atrophy (CCMA) is associated with mitochondrial injury. Mitochondrial calpains have been reported to induce mitochondrial injury in mouse cardiomyocytes and pulmonary smooth muscle. In the present study, the presence of calpain in the mitochondria of skeletal muscle and its potential role in CCMA were investigated. Transwell plates were used to develop a myotube-carcinoma cell co-culture model to simulate the cancer cachexia environment in vitro. The calpain inhibitors, calpastatin (CAST) and calpeptin (CAPT), were used to inhibit calpain activity in myotubes during co-culture. Calpain-1, calpain-2 and CAST were found to be present in mouse myotube mitochondria. Co-culture activated calpain in both cytoplasm and mitochondria, which caused myotube atrophy. CAST and CAPT treatment prevented calpain activation in both cytoplasm and mitochondria, which inhibited myotube atrophy during co-culture. Additionally, CAST and CAPT treatment increased mitochondrial complex I activity, decreased mitochondrial permeability transition pore opening and improved mitochondrial membrane potential in myotubes
\end{abstract}

Correspondence to: Dr Sizeng Chen, Department of Gastrointestinal Surgery, The First Affiliated Hospital of Fujian Medical University, 20 Chazhong Road, Fuzhou, Fujian 350004, P.R. China

E-mail: xiaotaiye@fjmu.edu.cn

Dr Xiantao Li, Department of Vascular and Endovascular Surgery, The First Affiliated Hospital of Fujian Medical University, 20 Chazhong Road, Fuzhou, Fujian 350004, P.R. China

E-mail: 1xtfreedom@126.com

Abbreviations: 4EBP1, eukaryotic translation initiation factor 4Ebinding protein 1; AIF, apoptosis-inducing factor; CAPT, calpeptin; CAST, calpastatin; CCMA, cancer cachectic muscle atrophy; CYCD, cyclophilin D; MHC, myosin heavy chain; MPTP, mitochondrial permeability transition pore; $\Delta \psi_{\mathrm{m}}$, mitochondrial membrane potential

Key words: cancer cachexia, calpain, mitochondria, muscle atrophy, co-culture during co-culture. In addition, CAST and CAPT treatment increased AKT/mTOR activity, inhibited FoxO3a activity and decreased atrogin-1 content in myotubes during co-culture. The present findings provide new insights to understand the mechanism of CCMA and further help the development of focused approaches to treat CCMA by manipulating the mitochondrial and cytosolic calpain activity.

\section{Introduction}

Cancer cachectic muscle atrophy (CCMA) is a multifactorial metabolic syndrome characterized by muscle atrophy and a progressive loss of muscle function (1). It occurs in $\sim 85 \%$ of terminal patients with upper gastrointestinal cancer and was responsible for $20 \%$ of all cancer deaths worldwide in 2010 (2). Conventional nutritional support cannot fully reverse CCMA, and no effective treatment has been reported (3). Although the pathogenesis of CCMA has been constantly researched in the past decade, it remains not well established.

CCMA is associated with increased muscle proteolysis and decreased protein synthesis, which involve the calpain system (4), the ubiquitin-proteasome system (5), the autophagy-lysosome system (6) and the PI3K/AKT signaling pathway (7). Since only the calpain system can degrade intact myofilaments, calpain-dependent cleavage of myofilaments is considered as the initial step in muscle proteolysis, serving a critical role in CCMA (8).

Calpains are a family of 15 calcium-activated cysteine proteases, including two ubiquitously expressed members, calpain-1 and calpain-2, and one muscle-specific member, calpain-3 $(9,10)$. Calpastatin (CAST) is the only known ubiquitously expressed endogenous calpain inhibitor (9). Calpains and CAST are generally considered to be localized in the cytoplasm and involved in numerous physiological and disease processes, including muscular dystrophy, diabetes, neurological disorders and hematonosis $(11,12)$. Calpain-1, calpain-2 and CAST have been found in the mitochondria of cardiomyocytes and pulmonary smooth muscle cells; in these cells, activation of mitochondrial calpain induces mitochondrial injury and cell damage $(13,14)$.

It has been reported that CCMA is also associated with mitochondrial injury (15). To the best of our knowledge, the role of mitochondrial calpain in CCMA has not yet been investigated. In the present study, a Transwell-plate system was 
used to develop a myotube-carcinoma cell co-culture model to simulate the cancer cachexia environment in vitro. The presence of calpains in the mitochondria of skeletal muscle and their potential role in CCMA were investigated.

\section{Materials and methods}

Cell culture. Mouse C2C12 myoblasts and CT26 colon carcinoma cells were obtained from the American Type Culture Collection and maintained in DMEM (Invitrogen; Thermo Fisher Scientific, Inc.) supplemented with $10 \%$ fetal bovine serum (Gibco; Thermo Fisher Scientific, Inc.) and 1\% penicillin-streptomycin. The cells were cultured in an atmosphere of $5 \% \mathrm{CO}_{2}$ at $37^{\circ} \mathrm{C}$.

To establish the co-culture system, $\mathrm{C} 2 \mathrm{C} 12$ myoblasts were seeded in 6-well plates at a density of 30,000 cells $/ \mathrm{cm}^{2}$ and cultured in growth medium for $24-48 \mathrm{~h}$ at $37^{\circ} \mathrm{C}$ to reach 90-100\% confluence. Subsequently, the growth medium was replaced by differentiation medium composed of DMEM and 2\% horse serum (Gibco; Thermo Fisher Scientific, Inc.) to induce myoblast differentiation. The myoblasts were cultured for another 4 days at $37^{\circ} \mathrm{C}$ to allow their differentiation into myotubes, and the differentiation medium was replaced by growth medium. At the same time, CT26 cells were seeded $\left(20,000\right.$ cells $\left./ \mathrm{cm}^{2}\right)$ into Transwell inserts (Corning Life Sciences) on a different 6-well plate containing growth medium. After a further $24 \mathrm{~h}$ of culture at $37^{\circ} \mathrm{C}$, the inserts were placed into the wells containing myotubes, and the medium was changed to growth medium with or without the calpain inhibitors CAST (cat. no. 208902; $1 \mu \mathrm{M}$; EMD Millipore) and calpeptin (CAPT; cat. no. C8999; $50 \mu \mathrm{M}$; Sigma-Aldrich; Merck KGaA). After $24 \mathrm{~h}$ of co-culture at $37^{\circ} \mathrm{C}$, the myotubes were fixed for immunocytochemistry or harvested for further analysis.

The co-culture combinations consisted of 5 groups: i) Sham myotubes (without CT26 cells in the insert) without calpain inhibitors (NC group); ii) myotubes co-cultured with CT26 cells but without calpain inhibitors (CO group); iii) myotubes with CT26 cells and CAST (CAST group); iv) myotubes with CT26 cells and CAPT (CAPT group); and v) myotubes with CT26 cells and CAST plus CAPT (CC group).

Immunocytochemical analysis. Myotubes were fixed, permeabilized, blocked and incubated with anti-myosin heavy chain (MHC) primary antibody (1:200; cat. no. ab91506; Abcam) followed by Alexa Fluor 488-conjugated goat anti-rabbit IgG secondary antibody (1:500; cat. no. ab150077; Abcam) as previously described (16). The myotubes were then mounted with Fluoroshield Mounting Medium containing DAPI (cat. no. ab104139; Abcam). Images were acquired with a fluorescence microscope (Nikon Corporation; magnification, x100) and analyzed with ImageJ software (version 1.46r; National Institutes of Health).

Myotube diameters were measured as previously described (17). Briefly, 20 images/well were captured, and the diameters of the five largest myotubes (those containing $\geq 3$ nuclei when viewed at x100 magnification) in each image were measured. Next, the mean diameter of a single myotube was calculated based on three independent measurements. The measurement points were separated by $200 \mu \mathrm{m}$. This method was also used to calculate the mean diameter \pm SD of the 100 largest myotubes in each well.

Isolation of myotube mitochondria. The Mitochondria Isolation kit for Cultured Cells (cat. no. ab110170; Abcam) was used to isolate myotube cytoplasm and mitochondria according to the manufacturer's protocol as previously described (18). Both cytoplasm and mitochondria were collected for further analysis.

Western blot analysis. Myotube cytosolic and mitochondrial proteins were extracted and separated via SDS-PAGE, blocked and stained with primary antibodies and HRP-conjugated secondary antibodies (1:2,000; cat. nos. ab97051 and ab6789; Abcam), and visualized as previously described (19). Images of the membranes were recorded with ChemiDoc XRS+ system (Bio-Rad Laboratories, Inc.) and analyzed using Quantity One software (version 4.6.6; Bio-Rad Laboratories, Inc.). The following primary antibodies were used: MHC $(1: 1,000$; cat. no. ab124937), calpain-1 (1:1,000; cat. no. ab108400), calpain-2 (1:2,000; cat. no. ab126600), CAST (1:1,000; cat. no. ab28252), NADH dehydrogenase (ubiquinone) iron-sulfur protein 3, mitochondrial (NDUFS3; 1:2,000; cat. no. ab177471), cyclophilin D (CYCD; 1:2,000; cat. no. ab181983), phosphorylated- (p-) AKT S473 (1:1,000; cat. no. ab81283), AKT (1:5,000; cat. no. ab179463), p-mTOR S2448 (1:1,000; cat. no. ab109268), mTOR (1:1,000; cat. no. ab32028), p-eukaryotic translation initiation factor 4E-binding protein 1 (p-4EBP1) T37 (1:1,000; cat. no. ab75767), 4EBP1 (1:2,000; cat. no. ab32024), p-FoxO3a S253 (1:1,000; cat. no. ab31109), FoxO3a (1:1,000; cat. no. ab70315), atrogin1 (1:2,000; cat. no. ab168372), muscle-specific RING finger protein 1 (MuRF1; 1:2,000; cat. no. ab172479), GAPDH (1:2,000; cat. no. ab181602) and voltage-dependent anion-selective channel protein 1 (VDAC; 1:1,000; cat. no. ab154856), all purchased from Abcam; calpain-3 (1:1,000; cat. no. sc-365277) and spectrin [cat. no. sc-46696; detects both full-length $(\mathrm{F})$-spectrin and cleaved (C)-spectrin] from Santa Cruz Biotechnology, Inc.; and apoptosis-inducing factor (AIF; 1:1,000; cat. no. 4642S) from Cell Signaling Technology, Inc.

Calpain activity assay. A calpain activity assay kit (cat. no. QIA120; EMD Millipore) was used to measure calpain activity in samples of myotube cytoplasm and mitochondria, according to the manufacturer's protocol as previously described (20). The assay plates were read using a SpectraMax M5 microplate reader with a wavelength of $380 \mathrm{~nm}$ (Molecular Devices, LLC).

Complex I enzyme activity assay. The Complex I Enzyme Activity Microplate Assay kit (cat. no. ab109721; Abcam) was used to measure mitochondrial complex I enzyme activity in samples of myotube mitochondria according to the manufacturer's protocol as previously described (18). The assay plates were read using a SpectraMax M5 microplate reader with a wavelength of $450 \mathrm{~nm}$.

JC-1 staining assay. Myotubes from 2 wells were collected using trypsin (cat. no. 25200072; Gibco; Thermo Fisher Scientific, Inc.) and plated in 96-well dark plates. JC-1 dye (cat. 


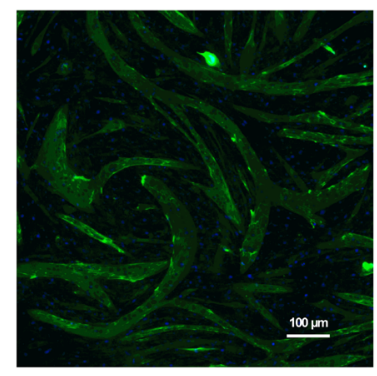

NC

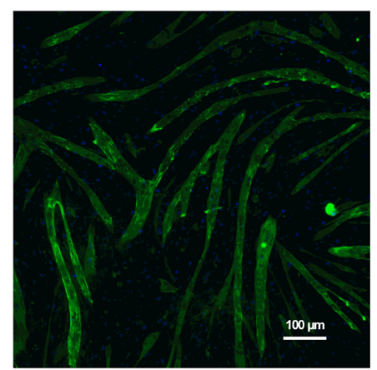

CAPT

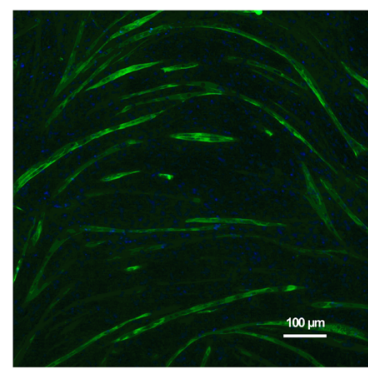

CO

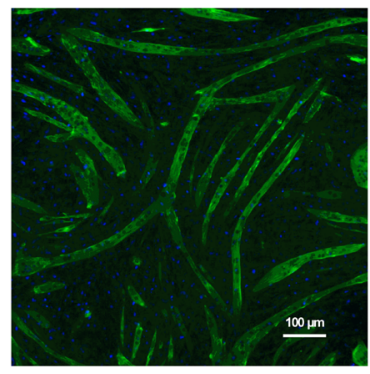

CC

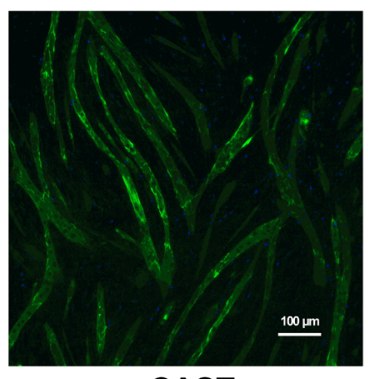

CAST

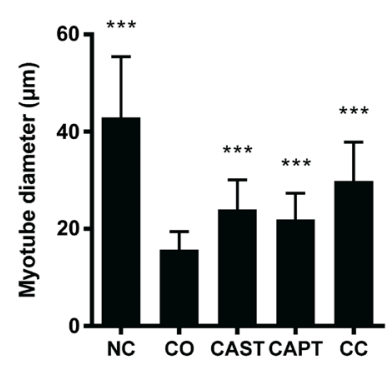

Figure 1. Effect of calpain inhibitors in myotube atrophy. Immunofluorescence staining for anti-MHC antibody in mouse C2C12 myotubes. MHC staining outlines the myotubes (green). DAPI was used to stain nuclei (blue). Magnification, x100. Scale bar, $100 \mu \mathrm{m}$. The myotube diameter was measured for each group and is represented in the bar graph. Data are represented as mean $\pm \mathrm{SD}$. ${ }^{* * *} \mathrm{P}<0.001$ vs. CO. MHC, myosin heavy chain; NC group, sham myotubes without CT26 cells and without calpain inhibitors; CO group, myotubes with CT26 cells without calpain inhibitors; CAST group, myotubes with CT26 cells and CAST; CAPT group, myotubes with CT26 cells and CAPT; CC group, myotubes with CT26 cells and CAST plus CAPT; CAST, calpastatin; CAPT, calpeptin.

no. C2006; Beyotime Institute of Biotechnology) was used to detect the mitochondrial membrane potential $\left(\Delta \psi_{\mathrm{m}}\right)$ according to the manufacturer's protocol. $\Delta \psi_{\mathrm{m}}$ was measured by quantifying the fluorescence emission shift from green $(530 \mathrm{~nm})$ monomers to red $(590 \mathrm{~nm})$ aggregates $(21)$. The assay plates were read using a SpectraMax M5 microplate reader. Data were expressed as fold increase in the red/green ratio.

Mitochondrial permeability transition pore (MPTP) opening. Myotubes were collected and plated in 96-well dark plates. MitoProbe Transition Pore Assay kit (cat. no. M34153; Thermo Fisher Scientific, Inc.) was used to measure MPTP opening according to the manufacturer's protocol as previously described (21). Myotubes were incubated with calcein AM dye (included in the kit) for $15 \mathrm{~min}$ and then incubated with $\mathrm{CoCl} 2$ for $15 \mathrm{~min}$ at $37^{\circ} \mathrm{C}$. Ionomycin was used as a positive control. The assay plates were read using a SpectraMax M5 microplate reader with a wavelength of $480 \mathrm{~nm}$.

Statistical analysis. Each experiment was repeated at least three times, and all data were analyzed using SPSS version 19.0 (IBM Corp.). Results are shown as the mean \pm SD. Statistical comparisons between groups were analyzed using one-way ANOVA followed by Tukey's post-hoc test when equal variances were assumed or Dunnett's T3 test when equal variances were not assumed. $\mathrm{P}<0.05$ was considered to indicate a statistically significant difference.

\section{Results}

Calpain inhibitors decrease myotube atrophy. Myotube diameter was significantly decreased following $24 \mathrm{~h}$ of co-culture (CO group) compared with the NC group (Fig. 1), suggesting that the cell co-culture model can simulate muscle atrophy. Compared with untreated myotubes (CO group), both CAST and CAPT treatment, as well as the combination of both treatments (CC group), significantly increased myotube diameter, indicating that inhibition of calpain attenuated myotube atrophy during co-culture of myoblasts and colon carcinoma cells.

Calpain inhibitors prevent calpain activation during co-culture. Co-culture activated both cytosolic and mitochondrial calpains in myotubes. Both cytosolic calpain-1 (cyt-CPN1) and mitochondrial calpain-1 (mit-CPN1) contents were increased by co-culture compared with the NC group (Fig. 2A, B and I). Direct enzyme activity assays revealed that cytosolic and mitochondrial calpain activities were also increased by co-culture (Fig. 2L and M). Cytosolic calpain activation can also be measured by calculating the $\mathrm{C}$-spectrin/F-spectrin ratio in myotubes (22). The results indicated that this ratio was significantly increased in co-cultured myotubes, indicating the increased formation of C-spectrin (Fig. 2A and F-H). Compared with the $\mathrm{CO}$ group, CAST, CAPT and CC treatment markedly decreased both CPN-1 expression in the western blots (Fig. 2A and B) and cytosolic calpain activity (Fig. 2L), the formation of C-spectrin (Fig. 2F-H) and the mit-CPN1 content (Fig. 2A and I). Cyt-CPN1 content (Fig. 2A and B) and mitochondrial calpain activity (Fig. 2M) were also decreased by CAST, CAPT and CC treatment, but did not reach a statistically significant difference. These data indicated that CAST, CAPT and CC treatment attenuated cytosolic and mitochondrial calpain activation. Cyt-CPN-2, cyt-CPN3, cyt-CAST, mit-CPN-2 and mit-CAST were also detected in myotubes, but their protein expression levels 

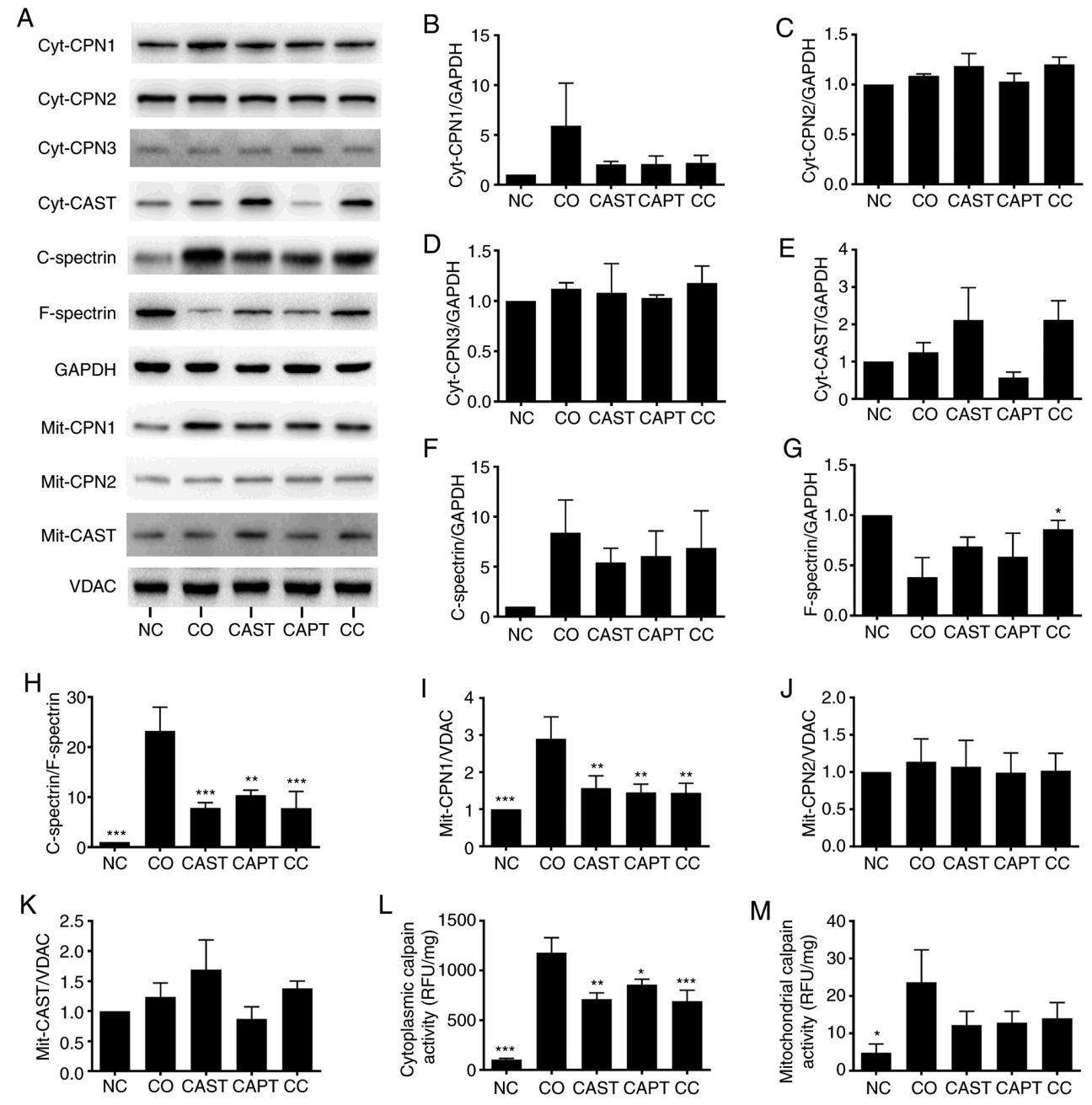

Figure 2. Effect of calpain inhibitors in the activation of calpain during co-culture. (A) Western blot results. Quantification of (B) cyt-CPN1, (C) cyt-CPN2, (D) cyt-CPN3 and (E) cyt-CAST normalized to GAPDH. (F) Quantification of cytosolic C-spectrin normalized to GAPDH. (G) Quantification of cytosolic F-spectrin normalized to GAPDH. (H) Quantification of C-spectrin/F-spectrin ratio. (I) Quantification of mit-CPN1 normalized to VDAC. Quantification of (J) mit-CPN2 and (K) mit-CAST normalized to VDAC. (L) Quantification of cytosolic calpain activity. (M) Quantification of mitochondrial calpain activity. Data are represented as the mean $\pm \mathrm{SD} .{ }^{*} \mathrm{P}<0.05,{ }^{* *} \mathrm{P}<0.01$ and ${ }^{* * * *} \mathrm{P}<0.001$ vs. CO. C-spectrin, cleaved spectrin; F-spectrin, full-length spectrin; $\mathrm{NC}$ group, sham myotubes without CT26 cells and without calpain inhibitors; CO group, myotubes with CT26 cells without calpain inhibitors; CAST group, myotubes with CT26 cells and CAST; CAPT group, myotubes with CT26 cells and CAPT; CC group, myotubes with CT26 cells and CAST plus CAPT; CAST, calpastatin; CAPT, calpeptin; cyt-, cytosolic; CPN, calpain; VDAC, voltage-dependent anion-selective channel protein 1; mit-, mitochondrial; RFU, relative fluorescence unit.

were not altered by co-culture, CAST, CAPT or CC treatment (Fig. 2A, C-E, J and K).

Calpain inhibitors improve complex I activity in myotube mitochondria following co-culture. Complex I is the first respiratory complex of the mitochondrial electron transport chain (13). Activation of mitochondrial calpain damages complex I activity, which impairs energy generation, leads to MPTP opening and cardiac injury during reperfusion (13). Complex I activity was significantly decreased in mitochondria following co-culture compared with the NC control (Fig. 3A). CAST, CAPT and CC treatment slightly improved complex I activity, but did not reach statistical significance (Fig. 3A). Additionally, NDUFS3 is a core subunit of complex I that is essential for NADH oxidation and subsequent electron transfer through the complex, maintaining the activity of complex I (13). NDUFS3 content was not altered by co-culture, CAST, CAPT or CC treatment (Fig. 3B and C).
Calpain inhibitors increase the $\Delta \psi_{m}$ in myotubes following co-culture. JC-1 was used to assess the $\Delta \psi_{\mathrm{m}}$ in myotubes (21). The $\Delta \psi_{\mathrm{m}}$ was reflected by the JC-1 red/green ratio. Compared with the $\mathrm{NC}$ group, the JC-1 red/green ratio in the $\mathrm{CO}$ group was significantly decreased by $70 \%$ (Fig. 3D), indicating damage of the $\Delta \psi_{\mathrm{m}}$ following co-culture. However, treatment with CAST, CAPT or CC significantly improved this ratio compared with the $\mathrm{CO}$ group (Fig. 3D), indicating that the activation of calpain during co-culture is able to damage the $\Delta \psi_{\mathrm{m}}$ in myotubes.

Calpain inhibitors decrease the MPTP opening in myotube following co-culture. The opening of the MPTP results in a permeation of the mitochondrial membrane that leads to the loss of $\Delta \psi_{\mathrm{m}}$ (21). Thus, the MitoProbe assay was used to assess the MPTP opening in myotubes (21). The MPTP opening was significantly increased in the CO group compared with in the NC group (Fig. 3E), indicating the increased MPTP opening 

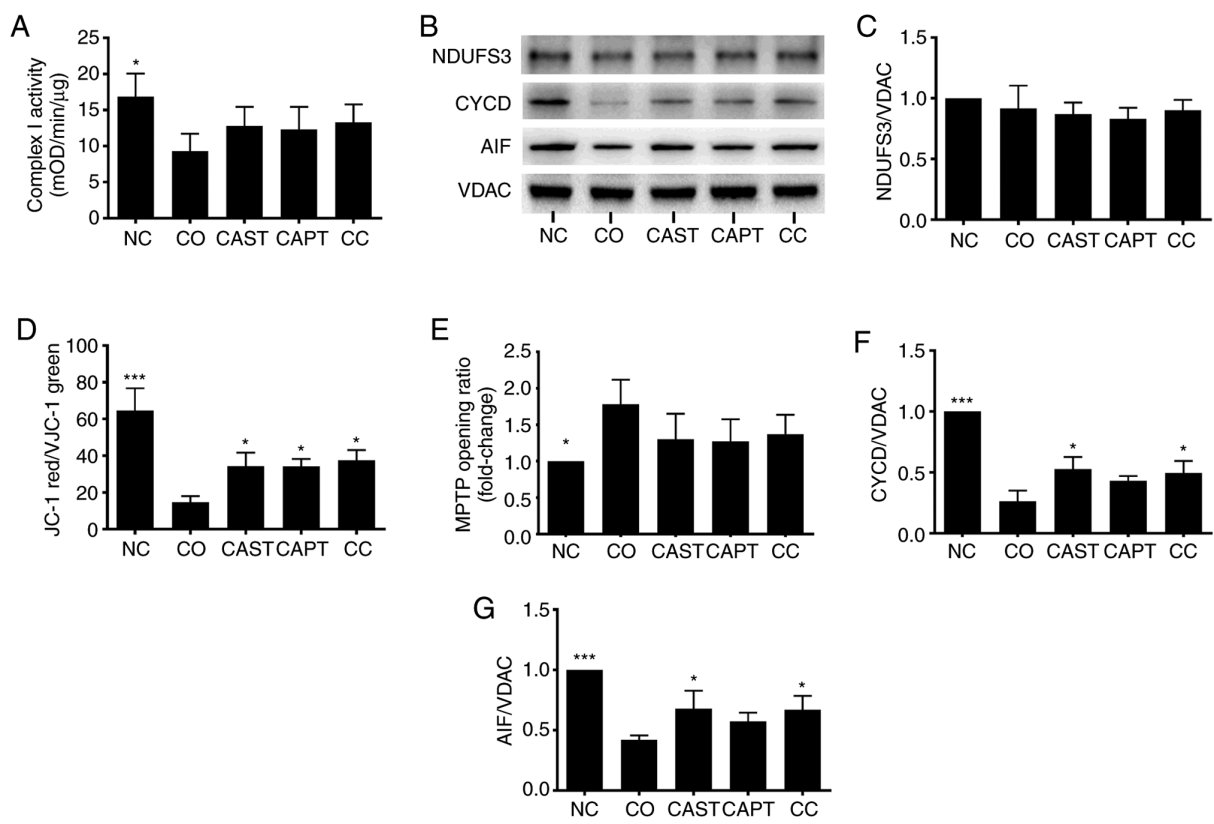

Figure 3. Effect of calpain inhibitors on complex I activity and MPTP opening in myotubes during co-culture with colon carcinoma cells. (A) Quantification of mitochondrial complex I activity. (B) Western blot results. (C) Quantification of mitochondrial NDUFS3 normalized to VDAC. (D) Quantification of JC-1 red/green ratio. (E) Quantification of MPTP opening ratio. (F) Quantification of mitochondrial CYCD normalized to VDAC. (G) Quantification of mitochondrial AIF normalized to VDAC. Data are represented as the mean $\pm \mathrm{SD}$. ${ }^{*} \mathrm{P}<0.05$ and ${ }^{* * * *} \mathrm{P}<0.001$ vs. CO. NC group, sham myotubes without CT26 cells and without calpain inhibitors; CO group, myotubes with CT26 cells without calpain inhibitors; CAST group, myotubes with CT26 cells and CAST; CAPT group, myotubes with CT26 cells and CAPT; CC group, myotubes with CT26 cells and CAST plus CAPT; CAST, calpastatin; CAPT, calpeptin; VDAC, voltage-dependent anion-selective channel protein 1; NDUFS3, NADH dehydrogenase (ubiquinone) iron-sulfur protein 3, mitochondrial; AIF, apoptosis-inducing factor; CYCD, cyclophilin D; MPTP, mitochondrial permeability transition pore; mOD, mean optical density.

A

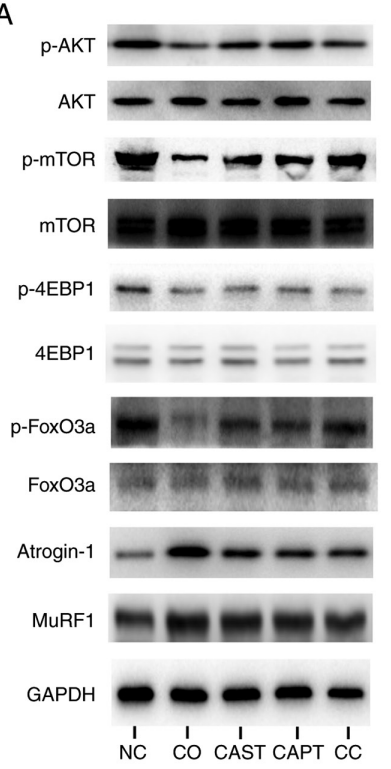

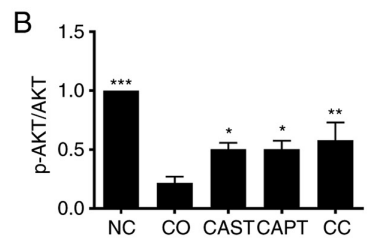

D
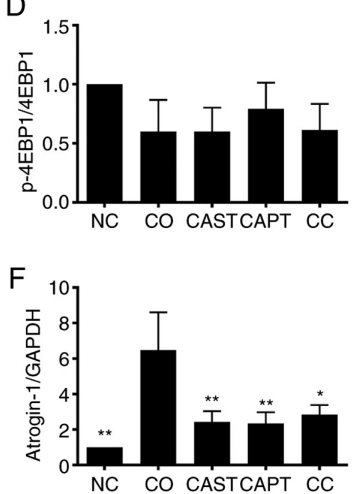

C

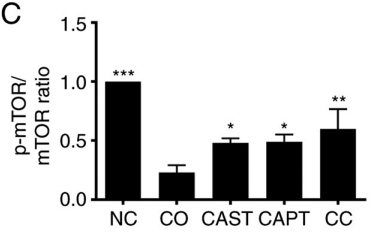

E

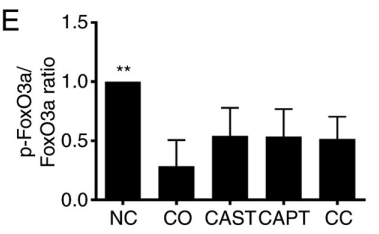

G

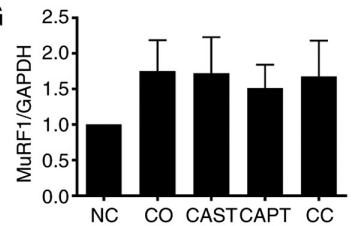

Figure 4. Effect of calpain inhibitors in AKT/FoxO3a signaling pathway activity and atrogin-1 content in myotubes during co-culture with colon carcinoma cells. (A) Western blot results. (B) Quantification of p-AKT/AKT ratio. (C) Quantification of p-mTOR/mTOR ratio. (D) Quantification of p-4EBP1/4EBP1 ratio. (E) Quantification of p-FoxO3a/FoxO3a ratio. (F) Quantification of atrogin-1 normalized to GAPDH. (G) Quantification of MuRF1 normalized to GAPDH. Data are represented as the mean $\pm \mathrm{SD}$. ${ }^{*} \mathrm{P}<0.05,{ }^{* *} \mathrm{P}<0.01$ and ${ }^{* * * *} \mathrm{P}<0.001$ vs. CO. p-, phosphorylated; NC group, sham myotubes without CT26 cells and without calpain inhibitors; CO group, myotubes with CT26 cells without calpain inhibitors; CAST group, myotubes with CT26 cells and CAST; CAPT group, myotubes with CT26 cells and CAPT; CC group, myotubes with CT26 cells and CAST plus CAPT; CAST, calpastatin; CAPT, calpeptin; 4EBP1, eukaryotic translation initiation factor 4E-binding protein 1; MuRF1, muscle-specific RING finger protein 1.

following co-culture. However, CAST, CAPT and CC treatment seemed to decrease the MPTP opening compared with the $\mathrm{CO}$ group, although there were no significant differences (Fig. 3E).
CYCD is a key factor that regulates MPTP opening (23). Compared with the NC group, co-culture significantly decreased the CYCD content in myotube mitochondria (Fig. 3B and F), whereas CAST and CC treatment significantly 
increased the CYCD content compared with co-culture alone. The present results suggested that the activation of calpain during co-culture decreased the CYCD content, which led to MPTP opening in mitochondria.

Calpain inhibitors preserve AIF content in myotube mitochondria. In addition to promoting the MPTP opening, calpain can cleave AIF and induce its release from the mitochondria to the cytoplasm $(24,25)$. Similarly, the current results demonstrated that co-culture significantly decreased the AIF content in myotube mitochondria compared with the NC group, and that this decrease was significantly attenuated by CAST and CC treatment (Fig. 3B and G). However, assays failed to detect the cleaved AIF in both cytoplasm and mitochondria (data not shown).

Calpain inhibitors increase the AKT/mTOR activity and decrease FoxO3a activity in myotubes following co-culture. $\mathrm{AKT} / \mathrm{mTOR}$ and FoxO3a serve a critical role in regulating muscular protein metabolism (26). Foxo3a is a transcription factor that activates the expression of ubiquitin ligase Atrogin-1 and MuRF1; Akt can phosphorylate FoxO3a and decrease its transcriptional activity (26). Co-culture significantly decreased the ratios of p-AKT/AKT, p-mTOR/mTOR and p-FoxO3a/FoxO3a in myotubes compared with the NC control group (Fig. 4A-C and E). Treatment with CAST, CAPT and CC significantly increased $\mathrm{p}-\mathrm{AKT} / \mathrm{AKT}$ and $\mathrm{p}-\mathrm{mTOR} / \mathrm{mTOR}$ ratios compared with the $\mathrm{CO}$ group (Fig. 4A-C), but not the p-FoxO3a/FoxO3a ratio (Fig. 4E). The p-4EBP1/4EBP1 ratio was not significantly affected by co-culture or treatment with the calpain inhibitors CAST or CAPT (Fig. 4A and D). The present results suggested that activation of calpain during co-culture decreased AKT/mTOR activity and increased FoxO3a activity in myotubes.

Calpain inhibitors decrease atrogin-1 content in myotubes following co-culture. Atrogin-1 and MuRF1 are two muscle-specific ubiquitin ligases that drive muscle protein degradation (8). As shown in Fig. 4A and F, co-culture significantly increased atrogin-1 content in myotubes compared with the NC control group, whereas CAST, CAPT and CC treatment significantly ameliorated this change. However, MuRF1 content was not significantly affected by co-culture or treatment with the calpain inhibitors CAST or CAPT (Fig. 4A and G).

\section{Discussion}

Cancer cachexia is a multifactorial syndrome that affects $\sim 50-80 \%$ of patients with cancer, depending on the tumor type $(27,28)$. In patients with gastric or pancreatic cancer, the incidence is $>80 \%$, whereas $\sim 50 \%$ of patients with colon, lung or prostate cancer are affected, and $\sim 40 \%$ of patients with breast cancer or some leukemias develop the syndrome $(27,28)$. In the present study, calpain-1, calpain-2 and CAST were detected in mouse myotube mitochondria. In addition, the results revealed that co-culture of myoblasts with colon carcinoma cells activated calpain in myotube mitochondria, caused MPTP opening and $\Delta \psi_{\mathrm{m}}$ damage, which led to mitochondrial injury. Moreover, co-culture activated calpain in myotube cytoplasm, caused the deactivation of AKT/mTOR and the activation of FoxO3a/atrogin-1. Calpain inhibitors (CAST, CAPT or CC) prevented calpain activation in both cytoplasm and mitochondria during co-culture, accompanied by inhibition of $\Delta \psi_{\mathrm{m}}$ damage and atrogin-1 expression. Therefore, the present results indicated that calpain inhibitors protected the myotube by inhibiting both cytosolic and mitochondrial calpain activity.

CT26 colorectal adenocarcinoma cell conditioned medium is often used to induce myotube atrophy to simulate CCMA in vitro $(29,30)$. In addition, CT26 colorectal adenocarcinoma and BALB/c mice can be used to develop cancer cachectic tumor-bearing mice, as previously described (4). Thus, in the present study, CT26 cells were used to develop a cell co-culture model with myoblasts to simulate CCMA.

Complex I is the first respiratory complex of the mitochondrial electron transport chain (31). Activation of mitochondrial calpain damages complex I activity, which impairs energy generation, leads to MPTP opening and cardiac reperfusion injury $(13,31)$. In the present study, co-culture impaired complex I activity in myotube mitochondria, whereas calpain inhibitors slightly improved complex I activity, indicating that the activation of mitochondrial calpain impairs complex I activity, which may lead to MPTP opening and energy generation impairment.

The mechanisms of complex I damage involves complex I subunit damage and post-translational modifications (32). NDUFS3 is a core subunit of complex I that is essential for NADH oxidation and subsequent electron transfer through the complex, maintaining the activity of complex I (13). In the present study, NDUFS3 content in myotube mitochondria was not altered by co-culture or by CAST, CAPT and CC treatment, indicating that the decreased complex I activity was not due to altered NDUFS3 content. It has been reported that complex I activity can be damaged by a conformational change (induced by a sulfhydryl oxidation of the second cysteine residue in complex I) (33). Therefore, the decreased complex I activity upon co-culture in the current study may be due to this post-translational modification, although this requires further investigation.

The MPTP is a non-selective pore located on the membrane of the mitochondria (34). MPTP opening increases the permeability of the mitochondrial membrane and leads to the loss of $\Delta \psi_{\mathrm{m}}$ and to mitochondrial injury, which in turn causes CCMA (27). In mouse hearts, calpain inhibitors attenuate the ischemia-reperfusion and induce MPTP opening and $\Delta \psi_{\mathrm{m}}$ depolarization (20). Additionally, calpain inhibitors ameliorate the microcystin-LR-induced MPTP opening and $\Delta \psi_{\mathrm{m}}$ depolarization in cultured hepatocytes (35), suggesting that calpain activation contributes to MPTP opening and $\Delta \psi_{\mathrm{m}}$ depolarization. Consistent with the aforementioned findings, the results of the present study demonstrated that co-culture activated calpain in myotube mitochondria, causing MPTP opening and $\Delta \psi_{\mathrm{m}}$ depolarization. By contrast, administration of CAST, CAPT or CC ameliorated these changes, suggesting that the activation of mitochondrial calpain induces MPTP opening and $\Delta \psi_{\mathrm{m}}$ depolarization. Furthermore, co-culture decreased the CYCD content in myotube mitochondria, whereas treatment with calpain inhibitors improved CYCD content, suggesting 
that the activation of mitochondrial calpain may increase MPTP opening through the cleavage of CYCD. However, no cleaved band of CYCD was detected in myotubes.

AIF is a mitochondrial flavoprotein that functions as an antioxidant in the mitochondrial intermembrane space (11). Activation of mitochondrial calpain cleaves AIF to truncated AIF (t-AIF) and releases t-AIF from the mitochondria to the cytoplasm, allowing it to translocate to the nucleus, inducing DNA degradation and apoptosis $(13,36)$. In the present study, co-culture decreased AIF content in myotube mitochondria, which was then reversed by calpain inhibitors, suggesting that activation of mitochondrial calpain induced the loss of AIF in the mitochondria. However, t-AIF was not detected in myotubes in the present study, suggesting that co-culture may induce myotube apoptosis via other mechanisms, which should be further investigated in future studies.

AKT/mTOR and FoxO3a serve a critical role in regulating muscular protein synthesis and proteolysis (26). AKT phosphorylates mTOR to increase its activity and promote protein synthesis (37). Activated mTOR in turn phosphorylates 4EBP1 (a negative regulator of the eukaryotic translation initiation factor 4E) to inhibit its activity (38). Additionally, AKT phosphorylates FoxO3a, inhibiting its activation and nuclear entrance, which decreases the expression levels of atrogin-1 and MuRF1 $(6,26)$. In the present study, co-culture decreased AKT and mTOR activity and increased FoxO3a activity and atrogin-1 content in myotubes, whereas calpain inhibitors ameliorated these changes, suggesting that the activation of cytosolic calpain acted through AKT/mTOR and FoxO3a/atrogin-1 to disrupt muscular protein metabolism.

Activation of autophagy contributes to muscle wasting $(7,39)$. Therefore, future studies should investigate whether the autophagy-lysosome system may be involved in the atrophy of myotubes.

To the best of our knowledge, calpain-1, calpain- 2 and CAST were for the first time demonstrated to be present in mouse myotube mitochondria in the present study. Calpain inhibitors protected the myotubes during co-culture by inhibiting both cytosolic and mitochondrial calpain activity. CAST is an endogenous calpain inhibitor, whereas CAPT is a commonly used non-selective and reversible calpain inhibitor; however, they both have off-target effects $(9,40,41)$. Therefore, a genetic approach is required to further clarify the potential role of mitochondrial calpain in CCMA. Investigation of mitochondrial calpain will provide new insights to understand the mechanism of CCMA. The present results will further help to develop focused approaches to attenuate CCMA by manipulating the mitochondrial and cytosolic calpain activity.

\section{Acknowledgements}

Not applicable.

\section{Funding}

The present was supported by Joint Funds for The Innovation of Science and Technology, Fujian province (grant no. 2018Y9083).

\section{Availability of data and materials}

The datasets used and/or analyzed during the current study are available from the corresponding author on reasonable request.

\section{Authors' contributions}

$\mathrm{XZ}$ drafted the main manuscript and performed the main experiments. XL and SC conceived and designed the experiments. LZ helped with cell culture, western blotting and immunofluorescence staining experiments, and was responsible for analyzing the data. All authors read and approved the final manuscript.

\section{Ethics approval and consent to participate}

Not applicable.

\section{Patient consent for publication}

Not applicable.

\section{Competing interests}

The authors declare that they have no competing interests.

\section{References}

1. Fearon K, Strasser F, Anker SD, Bosaeus I, Bruera E, Fainsinger RL, Jatoi A, Loprinzi C, MacDonald N, Mantovani G, et al: Definition and classification of cancer cachexia: An international consensus. Lancet Oncol 12: 489-495, 2011.

2. Donohoe CL, Ryan AM and Reynolds JV: Cancer cachexia: Mechanisms and clinical implications. Gastroenterol Res Pract 2011: 601434, 2011

3. Yoshida T, Semprun-Prieto L, Sukhanov S and Delafontaine P: IGF-1 prevents ANG II-induced skeletal muscle atrophy via Aktand Foxo-dependent inhibition of the ubiquitin ligase atrogin-1 expression. Am J Physiol Heart Circ Physiol 298: H1565-H1570, 2010.

4. Lin XY and Chen SZ: Calpain inhibitors ameliorate muscle wasting in a cachectic mouse model bearing CT26 colorectal adenocarcinoma. Oncol Rep 37: 1601-1610, 2017.

5. Johns N, Stephens NA and Fearon KC: Muscle wasting in cancer. Int J Biochem Cell Biol 45: 2215-2229, 2013.

6. Tsubouchi H, Yanagi S, Miura A, Matsumoto N, Kangawa K and Nakazato M: Ghrelin relieves cancer cachexia associated with the development of lung adenocarcinoma in mice. Eur J Pharmacol 743: 1-10, 2014.

7. Penna F, Costamagna D, Pin F, Camperi A, Fanzani A, Chiarpotto EM, Cavallini G, Bonelli G, Baccino FM and Costelli P: Autophagic degradation contributes to muscle wasting in cancer cachexia. Am J Pathol 182: 1367-1378, 2013.

8. Goll DE, Neti G, Mares SW and Thompson VF: Myofibrillar protein turnover: The proteasome and the calpains. J Anim Sci 86 (Suppl): E19-E35, 2008

9. Dókus LE, Yousef M and Bánóczi Z: Modulators of calpain activity: Inhibitors and activators as potential drugs. Expert Opin Drug Discov 15: 471-486, 2020.

10. Huang J and Zhu X: The molecular mechanisms of calpains action on skeletal muscle atrophy. Physiol Res 65: 547-560, 2016.

11. Ozaki T, Tomita H, Tamai M and Ishiguro S: Characteristics of mitochondrial calpains. J Biochem 142: 365-376, 2007.

12. Goll DE, Thompson VF, Li H, Wei W and Cong J: The calpain system. Physiol Rev 83: 731-801, 2003.

13. Chen Q, Thompson J, Hu Y, Dean J and Lesnefsky EJ: Inhibition of the ubiquitous calpains protects complex I activity and enables improved mitophagy in the heart following ischemia-reperfusion. Am J Physiol Cell Physiol 317: C910-C921, 2019. 
14. Kar P, Chakraborti T, Roy S, Choudhury R and Chakraborti S: Identification of calpastatin and mu-calpain and studies of their association in pulmonary smooth muscle mitochondria. Arch Biochem Biophys 466: 290-299, 2007.

15. Tisdale MJ: Mechanisms of cancer cachexia. Physiol Rev 89: 381-410, 2009.

16. Zeng X, Chen S, Yang Y and Ke Z: Acylated and unacylated ghrelin inhibit atrophy in myotubes co-cultured with colon carcinoma cells. Oncotarget 8: 72872-72885, 2017.

17. Trendelenburg AU, Meyer A, Rohner D, Boyle J, Hatakeyama S and Glass DJ: Myostatin reduces Akt/TORC1/p70S6K signaling, inhibiting myoblast differentiation and myotube size. Am J Physiol Cell Physiol 296: C1258-C1270, 2009.

18. Zhang XL, Wang ZZ, Shao QH, Zhang Z, Li L, Guo ZY, Sun $\mathrm{HM}$, Zhang Y and Chen NH: RNAi-mediated knockdown of DJ-1 leads to mitochondrial dysfunction via Akt/GSK-3 $\beta$ and JNK signaling pathways in dopaminergic neuron-like cells. Brain Res Bull 146: 228-236, 2019.

19. Zeng X, Chen S, Lin Y and Ke Z: Acylated and unacylated ghrelin inhibit apoptosis in myoblasts cocultured with colon carcinoma cells. Oncol Rep 39: 1387-1395, 2018.

20. Thompson J, Hu Y, Lesnefsky EJ and Chen Q: Activation of mitochondrial calpain and increased cardiac injury: Beyond AIF release. Am J Physiol Heart Circ Physiol 310: H376-H384, 2016.

21. Hendriks KDW, Joschko CP, Hoogstra-Berends F, Heegsma J, Faber KN and Henning RH: Hibernator-derived cells show superior protection and survival in hypothermia compared to non-hibernator cells. Int J Mol Sci 9: 1864, 2020.

22. Kudo-Sakamoto Y, Akazawa H, Ito K, Takano J, Yano M, Yabumoto C, Naito AT, Oka T, Lee JK, Sakata Y, et al: Calpain-dependent cleavage of $\mathrm{N}$-cadherin is involved in the progression of post-myocardial infarction remodeling. J Biol Chem 289: 19408-19419, 2014

23. Fakharnia F, Khodagholi F, Dargahi L and Ahmadiani A: Prevention of cyclophilin D-mediated mPTP opening using cyclosporine-A alleviates the elevation of necroptosis, autophagy and apoptosis-related markers following global cerebral ischemia-reperfusion. J Mol Neurosci 61: 52-60, 2017.

24. Cao G, Xing J, Xiao X, Liou AK, Gao Y, Yin XM, Clark RS, Graham SH and Chen J: Critical role of calpain I in mitochondrial release of apoptosis-inducing factor in ischemic neuronal injury. J Neurosci 27: 9278-9293, 2007.

25. Polster BM, Basañez G, Etxebarria A, Hardwick JM and Nicholls DG: Calpain I induces cleavage and release of apoptosis-inducing factor from isolated mitochondria. J Biol Chem 280: 6447-6454, 2005.

26. Clavel S, Siffroi-Fernandez S, Coldefy AS, Boulukos K, Pisani DF and Dérijard B: Regulation of the intracellular localization of Foxo3a by stress-activated protein kinase signaling pathways in skeletal muscle cells. Mol Cell Biol 30: 470-480, 2010.

27. Argilés JM, Busquets S, Stemmler B and López-Soriano FJ: Cancer cachexia: Understanding the molecular basis. Nat Rev Cancer 14: 754-762, 2014.

28. Fearon KC, Glass DJ and Guttridge DC: Cancer cachexia: Mediators, signaling, and metabolic pathways. Cell Metab 16: 153-166, 2012.
29. Lokireddy S, Wijesoma I, Bonala S, Wei M, Sze S, McFarlane C, Kambadur R and Sharma M: Retraction: Myostatin is a novel tumoral factor that induces cancer cachexia. Biochem J 473: 1111,2016

30. Sun R, Zhang S, Hu W, Lu X, Lou N, Yang Z, Chen S, Zhang X and Yang H: Valproic acid attenuates skeletal muscle wasting by inhibiting C/EBP $\beta$-regulated atrogin 1 expression in cancer cachexia. Am J Physiol Cell Physiol 311: C101-C115, 2016.

31. Karamanlidis G, Lee CF, Garcia-Menendez L, Kolwicz SC Jr, Suthammarak W, Gong G, Sedensky MM, Morgan PG, Wang W and Tian R: Mitochondrial complex I deficiency increases protein acetylation and accelerates heart failure. Cell Metab 18: 239-250, 2013.

32. Hollander JM, Thapa D and Shepherd DL: Physiological and structural differences in spatially distinct subpopulations of cardiac mitochondria: Influence of cardiac pathologies. Am J Physiol Heart Circ Physiol 307: H1-H14, 2014.

33. Galkin A, Abramov AY, Frakich N, Duchen MR and Moncada S: Lack of oxygen deactivates mitochondrial complex I: Implications for ischemic injury? J Biol Chem 284: 36055-36061, 2009.

34. Halestrap AP: What is the mitochondrial permeability transition pore? J Mol Cell Cardiol 46: 821-831, 2009.

35. Ding WX, Shen HM and Ong CN: Calpain activation after mitochondrial permeability transition in microcystin-induced cell death in rat hepatocytes. Biochem Biophys Res Commun 291: 321-331, 2002.

36. Ye H, Cande C, Stephanou NC, Jiang S, Gurbuxani S, Larochette N, Daugas E, Garrido C, Kroemer G and Wu H: DNA binding is required for the apoptogenic action of apoptosis inducing factor. Nat Struct Biol 9: 680-684, 2002.

37. Glass DJ: Skeletal muscle hypertrophy and atrophy signaling pathways. Int J Biochem Cell Biol 37: 1974-1984, 2005.

38. Sandri M: Signaling in muscle atrophy and hypertrophy. Physiology (Bethesda) 23: 160-170, 2008.

39. Tardif N, Klaude M, Lundell L, Thorell A and Rooyackers O: Autophagic-lysosomal pathway is the main proteolytic system modified in the skeletal muscle of esophageal cancer patients. Am J Clin Nutr 98: 1485-1492, 2013.

40. Yoshida M, Miyasaka Y, Ohuchida K, Okumura T, Zheng B, Torata N, Fujita H, Nabae T, Manabe T, Shimamoto M, et al: Calpain inhibitor calpeptin suppresses pancreatic cancer by disrupting cancer-stromal interactions in a mouse xenograft model. Cancer Sci 107: 1443-1452, 2016.

41. Tsujinaka T, Kajiwara Y, Kambayashi J, Sakon M, Higuchi N, Tanaka T and Mori T: Synthesis of a new cell penetrating calpain inhibitor (calpeptin). Biochem Biophys Res Commun 153: 1201-1208, 1988 . 